\title{
Hepatite A: avaliação do custo-benefício da prevenção pela vacina - Paraná, Brasil
}

\section{Hepatitis A: an assessment of the cost-benefit ratio of prevention by the vaccine - Paraná, Brazil}

\author{
Mariana Ribas Zahdi? \\ Ivan Maluf Junior \\ Eliane Mara Cesario Pereira Maluf ${ }^{2}$
}

\section{Resumo}

Objetivos: conhecer a magnitude do problema da Hepatite A no Paraná, Brasil, e avaliar custo-beneficio da vacinação. Materiais e métodos: o estudo é descritivo e de farmacoeconomia. Para avaliar a magnitude do problema, foram coletados dados sobre os casos de hepatite A do Sistema de Informação de Agravos de Notificação, (SINAN), Sistema de Informação em Mortalidade (SIM) e Autorização de Internação Hospitalar (AIH) da Secretaria de Estado da Saúde do Paraná referente ao período de 2000 a 2003. Foi estimada a probabilidade de uma coorte de crianças adquirir Hepatite $A$ durante a vida e os custos dos tratamentos. Esses gastos foram comparados com o custo da vacinação. Resultados: foram 14.682 notificações das quais 12.102 (82,4\%) ocorreram em menores de 15 anos. A incidência na população foi de 37,5/100000. Ocorreram 20 óbitos, sendo sete por insuficiência hepática. O custo com a doença foi comprovadamente alto. $\mathrm{O}$ investimento para a vacinação de crianças, com duas doses (10 USD/dose), também foi estimado. Observou-se uma relação custo-benefício positiva da vacinação. Para cada dólar investido na vacinaçào, 2,26 USD são economizados no tratamento. Conclusões: os resultados confirmam a relação custo-beneficio positiva da vacina. Sendo esse o primeiro estudo farmacoeconômico sobre a vacinação contra hepatite $A$ em nosso meio, apontase para a necessidade de pesquisas mais aprofundadas e abrangentes, com o intuito de complementar alguns aspectos ainda não explorados.

\section{Abstract}

Objectives: know the magnitude of the problem of Hepatitis A in Paraná, Brazil, and assess the cost-bentefit ratio of vacination. Materials and methods: the study is descriptive and pharmacoeconomical. To assess the magnitude of the problem, data were collected on cases of bepatitis A from the Information System of Worsening Notification (SINAN), Information System in Mortality (SLM) and Hospitalization Authorization (AIH) of the State Secretariat of Health of Parana referring to the period from 2000 to 2003. The?

Palavras-chave: Hepatite A; Epidemiologia, Vacinas

Contra Hepatite A; Análise Custo-Benefício.
Key Words: Hepatitis A; Epidemiology, Vaccines against Hepatitis A; Cost-Benefit Analysis.

Académicos, $6^{\circ}$ ano do Curso de Medicina, Universidade Positivo, Curitiba, Paraná, Brasil.

Professora Doutora, curso de Medieina, Universidade Positivo, Universidade Federal do Paraná, Curitiba, Paraná, Brasil. 
probability of one tenth of children acquiring Hepatitis A during their lifetime was estimated along with the costs of the treatments. These expenses were compared with the cost of vaccination.

Results: there were 14,682 notices of which 12,102 (82.4\%) accurred in cbildren under 15 years of age. The incidence on the population was 37.5/100000. There were 20 deaths, being 7 dre to liver failure. Costs with the disease were truly high. The investment in children vaccination, with two doses (10 USD/dose), was also estimated. A positive cost-benefit relation of the vaccination was observed. For each dollar invested in vaccination, USS 2.26 are saved on the treatment.

Conclusions: the results confirm the positive cost-benefit relation of the vaccine. Since this is the first pharmacoeconomic study on vaccination against bepatitis $A$ in our environment, the need for more in-depth and encompassing studies is bighlighted, with the goal of complementing a fen aspects not explored yet.

\section{Introdução:}

A hepatite $\mathrm{A}$ è uma doença causada por um vírus $\mathrm{RNA}^{1,2}$. Em geral, é considerada benigna e autolimitada, raramente letal, e que não afeta outros órgãos. $\mathrm{O}$ quadro clínico é caracterizado por um estado agudo de febre, fadiga, desconforto abdominal, icterícia, a norexia e náuseas ${ }^{3}$. A taxa de letalidade, que aumenta com a idade, foi descrita como próxima de $0,3 \%$; no entanto, esta aumenta para $2 \%$ em pacientes com idade acima de 40 anos $^{2}$.

$O$ vírus é usualmente transmitido via fecal-oral, pelo contato interpessoal ou por meio de alimentos e água contaminados, condição comum em países em desenvolvimento 4 .

A população brasileira possui um risco alto de contrair a doença em decorrência do deficiente sistema de saneamento básico em algumas regiōes. A prevalência no país, determinada por testes sorológicos, é de $65 \%$. Mais especificamente, um estudo realizado no Estado do Rio de Janeiro com crianças entre 0 a 10 anos, revelou que $50 \%$ das de classe socioeconômica baixa possuíam anticorpos anti-HAV enquanto apenas $28 \%$ das pertencentes à classe média apresentavam anticorpos. Em Fortaleza, Ceará, mais de $90 \%$ das crianças de classe menos favorecida haviam sido infectadas enquanto a prevalência encontrada nos infantes de classe média e alta era inferior a $25 \%$. Existe uma alta prevalência de Hepatite A no norte e centro-oeste, semelhante a outros países em desenvolvimento, e uma prevalência decrescente no sudeste e sul. Esses fatos epidemiológicos sobre a doença no Brasil mostram que nas regiões mais desenvolvidas do país o número de crianças suscetíveis tem aumentado, em consequência de um melhor seneamento básico. Em decorrência, o número de adolescentes e adultos jovens não imunes também aumentou, ascendendo a taxa de incidência nessa idade. Alguns autores concordam que a vacinação nessas regiões é necessária devido ao fato de a doença ter maior gravidade em pacientes com idade mais avançada?

Como medida geral de prevenção, a implementação dos programas de saneamento básico deveria ser uma das ações principais".

O método mais efetivo na prevenção da doença atualmente é a aplicação da vacina, que é preparada por meio da cultura de células do vírus causador de hepatite $A$. A soroconversão é rápida (aproximadamente 12 dias), portanto, a vacina pode ser administrada após um período curto de exposição ${ }^{8}$. Estudo de eficácia da vacina realizado entre crianças tailandesas vacinadas aos 8 meses de vida mostrou que $94 \%$ obtiveram soroconversão, enquanto que entre aquelas vacinadas aos 12 meses de idade, a soroconversão foi de $99 \%$. Estudos realizados na China têm demonstrado que a imunidade induzida pela vacina oferece proteção por longo período (25 anos) ${ }^{10}$.

Com a possibilidade da prevenção da hepatite A com a vacina, dá-se como importante a realização de estudos de custo-benefício das medidas preventivas. Sendo o Estado do Paraná uma das Unidades Federadas com um sistema de vigilância epidemiológica que tradicionalmente investiga todos os casos desse agravo, tendo por essa razào maior número de casos, são objetivos deste estudo: avaliar a magnitude do problema em nosso meio e comparar os custos da doença para a sociedade com o custo da imunização 
ativa contra a hepatite A. Desta forma, espera-se contribuir para o controle da doença no Estado.

\section{Materiais e Métodos:}

Trata-se de um estudo descritivo que inclui uma análise farmacoeconômica. A execução do projeto foi aprovada pelo Comitê de Ética da Secretaria de Estado da Saúde do Paraná.

- Coleta de dados:

Foi elaborado o instrumento (planilha) para a coleta de dados referentes aos casos de hepatite $\mathrm{A}$ das seguintes fontes: - AIH Is (Autorização de Internações Hospitalares) do Estado do Paraná, no periodo de quatro anos (2000 a 2003).

- SINAN - Sistema de Informação de Agravos de Notificação. A hepatite A é de notificação obrigatória desde 1972 no Paraná. O período estudado foi de 2000 a 2003. O SINAN registra casos atendidos tanto pelo Sistema Único de Saúde, quanto pelo sistema conveniado e privado. - SIM (Sistema de Informação de Mortalidade), Paraná, período de quatro anos (2000 a 2003).

- Realização de estimativas de custos com: exames laboratoriais, tratamento para casos de hepatite fulminante, transplante hepático, visitas médicas e vacinação contra hepatite A. Os custos foram baseados no valor de procedimentos médicos estabelecidos pelo Sistema de Saúde Pública Brasileiro.

- Logística e pessoal:

Os dados coletados foram registrados em planilha do programa Excel por dois estudantes de Medicina orientados pela coordenadora da pesquisa e assessorados pelos técnicos da Secretaria de Estado da Saúde - Paraná. A análise dos dados foi realizada utilizando-se o programa SPSS versão 10. A análise farmacoeconòmica foi feita baseada na experiência de profissionais de Buenos Aires, Argentina ${ }^{4}$.

\section{Resultados:}

I - A magnitude do problema das hepatites causadas pelo vírus $\mathrm{A}$ no Estado do Paraná

a- Internações por hepatite $\mathrm{A}$ durante os anos de $2000 \mathrm{a}$ 2003.

Analisando os dados obtidos das AIHs, que representa a população usuária do serviço público de saúde, encontrou-se um total de 432 indivíduos internados com diagnóstico de hepatite pelo vírus A (CID B15) e 2.326 com o diagnóstico de hepatite viral aguda não especificada (CID B19). Sendo que os exames laboratoriais para hepatite B e C eram realizados sistematicamente na investigação diagnóstica, foi suposto que esses 2.326 casos de hepatite viral aguda não especificada eram provavelmente causados pelo virus $A$, porém sem confirmação laboratorial.

b- Casos de hepatite A notificados no serviço de Vigilància Epidemiológica (SINAN), Paraná, de 2000 a 2003.

A tabela 1 mostra a incidência anual dos casos. Observou-se uma reduçào do número de notificaçoes nos últimos anos.

O total de casos de hepatite A registrados no periodo estudado foi de 14.682. Os casos registrados no SINAN

Tabela 1. Distribuiçâo de Casos de Hepatite A por ano de ocorrência, Paraná (2000 a 2003)

\begin{tabular}{ccc}
\hline Ano & N casos & $\%$ \\
2000 & 5620 & 38,3 \\
2001 & 4761 & 32,4 \\
2002 & 2472 & 16,8 \\
2003 & 1829 & 12.5 \\
Total & 14682 & 100,0 \\
\hline
\end{tabular}

Fonte: no SINAN, CIDS, Secretaria de Estado da Saúde do Paraná. 
sâo representativos de toda a população.

A incidência anual de casos de hepatite $A$ no Estado do Paraná foi de 37,5/100.000 habitantes/ano.

Observou-se um leve predomínio de casos de hepatite A no sexo masculino $(52,5 \%)$ e um aumento no número de casos notificados nos meses de março, abril e maio.

As regiões que mais registraram casos de hepatite $A$ no período foram Curitiba e região metropolitana, com $30,4 \%$ dos casos; são as regiões nas quais a concentração da população é maior.

A idade dos casos variou de seis meses a 89 anos, tendo como média o valor de 10,1 anos e mediana de oito. Oitenta e dois por cento dos casos pertenciam à faixa etária de até 15 anos.

A incidência de hepatite $\mathrm{A}$ na faixa etária menor de cinco anos foi de 122,5 casos por 100.000 habitantes/ano. Para a população de cinco até 15 anos, foi de $100,3 / 100.000$ habitantes/ano. Na faixa etária de 16 a 49 anos, foi de $1,9 \mathrm{e}$ nas idades maior ou igual a 50 anos de $2,31 / 100.000$ habitantes/ano.

c- Óbitos por hepatite A no Estado do Paraná, 2000 a 2003.

Das Declarações de Óbito, 20 foram registradas com a CID de hepatite $A$.

Do total de óbitos, $12(60 \%)$ eram do sexo feminino ( $p<0,01)$, e $50 \%$ ocorreram em menores de 15 anos de idade.

A principal causa de óbito foi hepatite fulminante, representando $35 \%$ das mortes.
Tabela 2. Distribuição da População do Paraná por faixa etária.

$\begin{array}{cr}\text { Idade } & \text { Total } \\ <\text { < } 1 \text { ano } & 177635 \\ 1 \text { a } 4 \text { anos } & 732001 \\ 5 \text { a } 9 \text { anos } & 947363 \\ 10 \text { a } 14 \text { anos } & 958834 \\ 15 \text { a } 19 \text { anos } & 972799 \\ 20 \text { a } 29 \text { anos } & 1711221 \\ 30 \text { a } 39 \text { anos } & 1549833 \\ 40 \text { a } 49 \text { anos } & 1164898 \\ >=50 \text { anos } & 1583381 \\ \text { Total } & 9797965\end{array}$

* $\mathrm{O}$ ano de 2002 foi usado por ser intermediário entre os anos pesquisados no SINAN. FONTE: DATASUS/MS

II - Estimativas de custos:

1. Custo da doença:

Para avaliar o custo da doença, criou-se um modelo que mostra a probabilidade de uma coorte de crianças, desde o nascimento até 50 anos de idade, adquirir a enfermidade. Com esses resultados, estimaram-se os custos com a doença.

De acordo com a tabela 2 , existem 177.635 crianças entre 0 e 12 meses no Paraná.

A preparação do modelo estatístico foi baseada nos dados referentes à taxa de incidência de hepatite $\mathrm{A}$ na população até os 50 anos de idade ${ }^{12}$.

Exemplo: no grupo de 10 a 14 anos, 35,81\% são sorologicamente positivos (total de 13.713 da população

Quadro 1 - Estudo realizado em São Paulo, Roberto Focaccia et al, $1998^{12}$

\begin{tabular}{lccc}
\hline Faixa etária & \% de soro + p/ hep A & N provável de infecções & N de casos sintomáticos \\
\hline 2 a 4 anos & 12,10 & 21.493 & 6.447 \\
5 a 9 anos & 28,09 & 28.403 & 14.021 \\
10 a 14 anos & 35,81 & 13.713 & 9.599 \\
15 a 17 anos & 56,16 & 36.145 & 25.301 \\
18 a 29 anos & 65,30 & 16.235 & 11.364 \\
30 a 39 anos & 85,89 & 36.415 & 25.490 \\
40 a 49 anos & 90,81 & 8.739 & 6.117 \\
\hline
\end{tabular}

Número total de casos esperados de hepatite (nascimento até 50 anos) 98.339

* Menores de 7 anos - $70 \%$ assintomáticos; maiores de 8 anos $-70 \%$ sintomáticos 
do Paraná dessa faixa etária). Desses, $70 \%$ são sintomáticos (9.599 sintomáticos).

\section{1a. Estratégia 1: não vacinar.}

Variáveis analisadas:

1) Número de casos: 98.339 (quadro 1).

2) Número de consultas ( 4 por paciente): 393.356 consultas.

3) Laboratório (hemograma, TGP - TGO, bilirrubinas total e frações, coagulograma) - três vezes durante a doença:

1.475.085 determinações.

4) IgM: 98.339 determinações.

5) Número de recaídas (10\%): 9.833 casos.

6) Determinação laboratorial nos casos de recaídas $(10 \%)$ : 147.495 determinações.

7) Hepatite fulminante (um para cada mil casos sintomáticos): 98 casos.

8) Número de transplantes hepáticos ( $80 \%$ dos casos de hepatite fulminante): 78 casos.

9) Número de retransplantes (20\%): 15 casos.

Custo em dólares:

1) Consulta em dólares (1,6 US\$/ consulta) = US\$ 629.369

2) Laboratório (hemograma, TGP - TGO, coagulograma, bilirrubina $=$ média de 3,18 US\$ por determinação igual a US\$ 4.690 .770

3) Consultas em casos de recaídas (39332 casos por 1,6 US\$) $=$ US\$ 62.931

4) Laboratório nas recaidas: (147495 determinações) $=$ USS 469.034

5) IgM para diagnóstico $=98339$ determinações $(6,16$ USS cada uma) $=$ USS 599.867

6) Hepatite fulminante: 98 casos (cada internação 120 US\$)

$=$ USS 11.760

7) Número de transplantes: 78 casos (cada transplante $=$ 17.299 US\$) $=$ US\$ 1.349 .322

8) Número de retransplantes (17.557 USS por caso) = 263.355 USS

9) Total: US\$ 8.076 .408

\section{1 b. Estratégia 2: vacinar.}

Custo da vacinação de uma coorte de 177.635 nascidos vivos (Tabela 2).

1) Custo da dose da vacina $=$ US\$ 10 (cada criança $=$ duas doses).

2) Doses de vacina - custo total $=$ USS 355.270 .

3) Consultas por eventos adversos (3\% dos vacinados) $=$ 10.658 consultas - USS 17.052 .

4) Total do custo universal das vacinas $=$ USS 3.569 .752 .

Comparando-se os valores estimados em gastos com a doença (estratégia 1) e o custo da vacinação (estratégia 2), observou-se que para cada dólar investido na vacina, poupa-se 2,26 dólares com a doença. Deve-se considerar que não foram inclúdos custos da profilaxia pós-exposição, medicamento pós-transplante, nem os óbitos pela heparite A.

\section{Discussāo}

A análise dos dados de hepatite $\mathrm{A}$ no estado do Paraná, nos anos de 2000 a 2003, mostrou que $82,4 \%$ dos casos e $50 \%$ dos óbitos ocorreram em menores de 15 anos, sendo a média da idade igual a 10 anos. Esses dados divergem daqueles apresentados em pesquisa realizada na Holanda, onde a média de idade dos casos foi 35 anos e $77,8 \%$ desses ocorreram acima dos 15 anos ${ }^{7}$. Provavelmente, o padrão de ocorrência da doença é diferente pelos diferentes níveis de desenvolvimento das regiỏes.

Embora a hepatite A seja doença de notificação obrigatória no Paraná, a possibilidade de sub-registro em nosso meio é demonstrada quando se leva em consideração dados de literatura que apontam a proporção de aproximadamente uma morte a cada mil casos da doença. No Paraná, foram registrados 20 óbitos entre 14.682 casos de hepatite A no período estudado. A diferença entre o número de casos esperado e o observado reforça a possibilidade de subnotificação da doença. As possíveis razões da subnotificação são várias: falta de confirmação etiológica, falha na notificação por parte dos profissionais de saúde, entre ou$\operatorname{tros}^{13}$.

Insuficiência hepática foi a principal causa de morte (35\%) por hepatite A no Paraná, concordando com dados da literatura internacional ${ }^{14}$. Segundo Ciocca $\mathrm{M}$ et al, em estudo realizado na Argentina, Brasil, Chile, Colòmbia, Costa 
Rica, e México, demontrou-se que dos pacientes entre $1 \mathrm{e}$ 20 anos internados com suspeita de insuficiência hepática aguda, $43 \%$ apresentaram sorologia para HAV aguda (IgM). Desses, $41 \%$ necessitaram de transplante hepático, demonstrando a importância dessa doença como causa da insuficiência hepática fulminante ${ }^{15}$.

Os resultados acima apresentados confirmam a magnitude do problema em nosso meio. Acrescentado à possibilidade de ocorrência de complicações, especialmente quando a infecção é adquirida em idade mais avançada, o risco de insuficiência hepática, a necessidade de transplante hepático, o custo da manutenção do paciente transplantado, a dor e o sofrimento dos familiares, fazem com que a adoção de medidas preventivas seja relevante.

$A$ vacina contra hepatite $A$ é a opção de prevençāo com resultado em curto prazo. Há consenso na literatura sobre a elevada eficácia desse imunobiológico ${ }^{9,10}$, porém não há publicação nacional até o momento avaliando a relação custo-benefício da sua utilização entre a população brasileira.

$\mathrm{O}$ uso de modelo matemático permitiu estimar o resultado da implantação da vacina contra hepatite A no calendário de imunização da criança, no Estado do Paraná, bem como o custo dessa ação.

Considerando que a população menor de 12 meses do Paraná é de 177.635, o modelo propõe a vacinação de todas essas crianças ao completarem um ano de idade.

Baseado na análise do custo da vacina (US\$10 por dose) e na necessidade de duas doses para a obtenção de imunidade por longo tempo, a estimativa de gastos para essa vacinação seria de US\$ 3.552.700. Adicionando a isso, uma incidência de $3 \%$ de eventos adversos à vacina que requerem monitoramento por profissional de saúde, o custo total seria US\$ 3.569 .752 .

Levando em consideração os casos de hepatite $A$ no Paraná, em pacientes com 50 anos ou menos, considerados os gastos do tratamento, o custo total foi US\$ 8.076.408. Portanto, de um ponto de vista financeiro, a vacinaçào é definitivamente vantajosa na profilaxia contra a infecção viral, além de prevenir danos sociais, evitar mortes e prevenir epidemias.

Isso quer dizer que se essa coorte de 177.635 crianças for vacinada contra hepatite $\mathrm{A}$ aos 12 meses de idade, com um custo de US\$ 3.552.700, elas não desenvolverão a doença ao longo da vida. O gasto de US\$ 8.076.408 com a doença deixaria de existir, além do benefício de evitar dor, sofrimento e complicações. Esse cálculo provavelmente está subestimado, pois não foram incluídos na estimativa os custos da profilaxia pós-exposição, medicamento póstransplante, nem os óbitos pela hepatite A.

Neste estudo não foi avaliado o beneficio da "imunidade de rebanho", mas Wasley et al ${ }^{16}$ e Dagan R et al ${ }^{17}$ demonstraram em estudos nos Estados Unidos e Israel que um declínio nas taxas de infecções por hepatite $\mathrm{A}$ em crianças deu-se pela vacinação obrigatória, com consequente redução da transmissibilidade a outros. Isso corrobora a hipótese de efeito importante da "imunidade de rebanho", que, se contabilizada, certamente apontará para um benefício ainda maior.

Ferreira et al ${ }^{18}$ já afirmavam, em 1996, que a incidência de hepatite A não seria reduzida somente vacinando grupos específicos no Brasil. O ideal seria adotar a vacinação no calendário vacinal infantil de rotina em maiores de 12 meses.

Embora vários estudos internacionais apontem para uma relação custo-benefício positiva da vacinação contra hepatite $A^{19}$, um estudo farmacoeconômico com uma amostra de crianças vacinadas de uma minoria étnica em Amsterdam não mostrou vantagem econômica na estratégia ${ }^{20}$. O'Connor et al ${ }^{21}$ demonstraram, em 1999, que a relação custo-benefício seria satisfatória se a dose da vacina tivesse o preço inferior a 7 dólares. Diel $\mathrm{R}^{22}$ constatou que a vacinação em adolescentes e crianças alemãs não é vantajosa do ponto de vista econômico, exceção para viajantes a países de alta prevalência.

A hepatite A também apresenta alta incidência no Chile, onde é considerada um problema de saúde pública. Nesse país, a vacinação da população representaria uma economia de aproximadamente 4 bilhões de dólares ${ }^{23}$. Nos Estados Unidos, Jacobs RJ et af $\mathbb{F}^{4}$ estudaram crianças vaci- 
nadas que viviam em áreas endêmicas. Para esses casos, foi mostrada uma relação custo-benefício positiva para a vacinação do ponto de vista econômico e social. Atualmente, a vacinação é recomendada nos Estados Unidos e em Israel ${ }^{17}$. Rosenthal $\mathrm{P}^{25}$ revisou alguns estudos sobre análise econômica da vacina contra hepatite $A$ e concluiu que o benefício econômico é semelhante aos das outras vacinas que já estão incluídas no programa de rotina para imunização infantil, além de ser economicamente aceitável para adolescentes e adultos suscetíveis à doença.

\section{Conclusão}

Os resultados deste estudo confirmam a relação custo-benefício positiva da vacinaçào de crianças contra hepatite A.

Ponderando que: a incidência de hepatite A é elevada no Paraná, a vacina proporciona resultado positivo em termos de proteção em curto prazo, e é menos dispendioso vacinar que arcar com os custos da doença, a vacinação de rotina deve ser fortemente considerada.

Sendo este o primeiro estudo de custo-efetividade da vacinação contra hepatite $A$ em nosso meio, aponta-se para a necessidade de pesquisas mais aprofundadas e abrangentes, com o intuito de complementar alguns aspectos ainda não explorados.

\section{Referências}

1. Paula VS, Baptista ML, Lampe E, Niel C, Gaspar AM. Characterization of hepatitis A virus isolates from subgenotypes IA and IB in Rio de Janeiro, Brazil. J Med Virol. 2002 Jan; 66 (suppl 1):22-7.

2. Valenzuela MTB. Vacunas anti hepatitis A: Estado del arte. Rev Chil Infect. 1999; 16(1):37 - 44.

3. American Academy of Pediatrics. Hepatitis A. In: Pickering LK, ed. Red Book: 2006 Report of the Committee on Infectious Diseases. 26th ed. Elk Grove Village, IL: American Academy of Pediatrics; 2006: 32635.

4. Sonder GJB, Van Steenbergen JE, Bovee LP, Peerbooms PG, Coutinho RA, Van DEN Hoek A. Hepatitis A virus immunity and seroconversion among contacts of acute hepatitis A patients in Amsterdam, 1996 - 2000: An evaluation of current prevention policy: Am J Public Health. 2004; 94(9):1620-6.

5. Almeida LM, Werneck GL, Cairncross S, Coeli CM, Costa MC, Coletty PE. The intensity of transmission of hepatitis $\mathrm{A}$ and heterogeneities in socio-environmental, risk factors in Rio de Janeiro, Brazil. Trans R Soc Trop Med Hyg. 2002; 96(6):605-10.

6. Guimaraens MA, Codeço CT. Experiments with mathematical models to simulate hepatitis A population dynamics under different levels of endemicity. Cad Saude Pública. 2005; 21(5):1531 - 9.

7. Pereira FE, Gonçalves CS. Hepatitis A. Rev Soc Bras Med Trop. 2003; 36(3):387-400.

8. Gendrel D. Hepatitis-A vaccination in children. Med Trop (Mars). 2004; 64(4):394-400.

9. Soogarun S, Wiwanitkit V. Vaccinating Thai adolescents against hepatitis A: is it cost- effective? Southeast Asian J Trop Med Public Health. 2002; 33 Suppl 3:145 - 8.

10. Yang CY, Lu CY, Lee CY, Shao PL, Wang CY, Wu TZ, et al. An open study of inactivated hepatitis A vaccine (VAQTA) in Taiwanese healthy adult volunteers: safety, tolerability, and immunogenicity: Nicrobiol Immunol Infect. 2004; 37(4):216-8. 11. Lopez E, Debbag R, Coudeville I, Baron-Papillon F, Armoni J. The cost-effectiveness of universal vaccination of children against hepatitis A in Argentina: results of a dynamic healtheconomic analysis. J Gastroenterol. 2007; 42(2):152 - 60.

12. Focaccia R, da Conceição OJ, Sette H Jr, Sabino E, Bassit L, Nitrini DR, et al. Estimated prevalence of viral hepatitis in the general population of the municipality of Sào Paulo, measured by a serologic surver of a stratified, randomized and residence-based population. Braz J Infect Dis. 1998; 2(6):269-84.

13. Lagos RZ, Potin M, Munoz A, Abrego P, San Martin OS, Ureta AM, et al. Anticuerpos séricos contra el virus hepatitis A en sujetos de nivel socioeconómico medio y bajo, en comunas urbanas de Santiago. Rev Med Chil. 1999; $127(4): 429-36$.

14. Andre F, Van Damme P, Safary A, Banatvala J. Inactivated 
hepatitis A vaccine: immunogenicity, efficacy, safety and review of official recommendations for use. Expert Rev Vaccines. 2002; 1(1):9-23.

15. Ciocca M, Moreira-Silva SF, Alegría S, Galoppo MC, Rutiman R, Porta G, et al. Hepatitis A as an etiologic agent of acute liver failure in Latin America. Pediatr Infect Dis J. 2007; 26(8):711-5.

16. Wasley A, Samandari T, Bell BP. Incidence of hepatitis A in the United States in the era of vaccination. JAMA. 2005; 294(2):194-201.

17. Dagan R, Leventhal A, Anis E, Slater P, Ashur Y, Shouval D. Incidence of hepatitis A in Israel following universal immunization of toddlers. JAMA. 2005; 294(2):202-10.

18. Ferreira CT, Pereira Lima J, Barros FC. Soroepidemiologia da hepatite $\mathrm{A}$ em dois grupos populacionais economicamente distintos de Porto Alegre. GED. 1996; 15(3):85 - 90.

19. Deneke MG, Arguedas MR. Hepatitis A and considerations regarding the cost-effectiveness of vaccination programs. Expert Rev Vaccines. 2003; 2(5):661-72.

20. Postma MI, Bos JM, Beutels P, Schilthuis H, van den Hoek JA. Pharmaco-economic evaluation of targeted hepatitis A vaccination for children of ethnic minorities in Amsterdam (The Netherlands). Vaccine. 2004; 22(15-16):1862 - 7.

21. O'Connor JB, Imperiale TF, Singer ME. CostEffectiveness Analysis of Hepatitis A Vaccination Strategies for Adults. Hepatology. 1999; 30(4):1077-81.

22. Diel R, Rappenhoner B, Schneider S. Cost-effectiveness of hepatitis A immunization of children and adolescents in Germany. Eur J Health Econ. 2001; 2: 96-103.

23. Valenzuela MT, Jacobs RJ, Arteaga O, Navarrete MS, Meyerhoff AS, Innis BL. Cost-effectiveness of universal childhood hepatitis A vaccination in Chile. Vaccine. 2005; 23(32):4110-9.

24. Jacobs RJ, Greenberg DP, Koff RS, Saab S, Meyerhoff AS. Regional variation in the cost effectiveness of childhood hepatitis A immunization. Pediatr Infect Dis J. 2003; $22(10): 904-14$.

25. Rosenthal P. Cost-effectiveness of hepatitis A vaccination in children, adolescents, and adults. Hepatology. 2003; $37(1): 44-51$.
Nota:

Agradecemos os profissionais do Centro de Informação e Diagnóstico em Saúde (CIDS) - Secretaria de Saúde do Estado do Paraná, pelo auxílio na coleta de dados, o Prof. Dr. Roberto Debbag, coordenador do Departamento de Infectologia Pediátrica do Hospital Juan Garrahan de Buenos Aires, Argentina, pelo auxilio na realização da análise farmacoeconômica do estudo.

\section{Endereço para correspondência:}

Av Silva Jardim, 2.833.

Curitiba - PR.

CEP: $80.240-020$

\section{Endereço eletrônico:}

eliane.cesario@yahoo.com.br 